\title{
The package HarmonicSums: Computer Algebra and Analytic aspects of Nested Sums
}

Jakob Ablinger*i

Research Institute for Symbolic Computation (RISC)

Johannes Kepler University Linz, Altenberger Straße 69, A-4040 Linz, Austria

E-mail: jablinge@risc.jku.at

\begin{abstract}
This paper summarizes the essential functionality of the computer algebra package HarmonicSums. On the one hand HarmonicSums can work with nested sums such as harmonic sums and their generalizations and on the other hand it can treat iterated integrals of the Poincaré and Chen-type, such as harmonic polylogarithms and their generalizations. The interplay of these representations and the analytic aspects are illustrated by concrete examples.
\end{abstract}

Loops and Legs in Quantum Field Theory

27 April 2014 - 02 May 2014

Weimar, Germany

\footnotetext{
* Speaker.

$\dagger$ This work has been supported in part by the Austrian Science Fund (FWF) grant SFB F50 (F5009-N15) and by the EU Network LHCPHENOnetPITN-GA-2010-264564.
} 


\section{Introduction}

This paper is dedicated to the presentation of the basic features of the computer algebra package HarmonicSums which was developed in [2] and [1] and which was afterwards extended and generalized. The package HarmonicSums was designed to support calculations with special nested objects arising in massive higher order perturbative calculations in renormalizable quantum field theories. On the one hand HarmonicSums can work with nested sums such as harmonic sums [15, 26] and their generalizations (S-sums [4, 22], cyclotomic harmonic sums [3], cyclotomic S-sums [1], binomial sums [5, 6, 7, 21, 27]) and on the other hand it can treat iterated integrals of the Poincaré and Chen-type, such as harmonic polylogarithms [23] and their generalizations (multiple polylogarithms [4], cyclotomic harmonic polylogarithms [3]). HarmonicSums provides functions to compute (generalizations of) the Mellin-transformation of these iterated integrals which leads to the nested sums and on the other hand inverse Mellin transforms of the nested sums can be computed. Harmonic.Sums offers commands that rewrite certain types of nested sums into expressions in terms of generalized S-sums and it can be used to derive algebraic and structural relations between the nested sums (compare [1, 2, 16, 17, 18, 19]) as well as relations between the values of the sums at infinity and connected to it the values of the iterated integrals evaluated at special constants (compare [1, 3, 4, 20]). In addition algorithms to compute series expansions (especially asymptotic expansions) of these nested objects are implemented. The package has already been used extensively, for example during the work on [10, 11, 12, 13, 14].

\section{The package HarmonicSums}

Note that this section contains a whole Mathematica session that runs throughout the sections. The inputs are given in the way how one has to type them into Mathematica and the outputs are displayed as Mathematica gives them back. We start the session by loading the package HarmonicSums:

$\ln [1]:=\ll$ HarmonicSums.m

HarmonicSums by Jakob Ablinger -RISC Linz- Version 1.0 (15/05/04)

\section{Definition of the Nested Sums}

In the package HarmonicSums harmonic sums, S-sums, cyclotomic harmonic sums and cyclotomic S-sums are denoted by the letter $\mathrm{S}$ as we can see in the following examples.

The command ToHarmonicSums Sum yields the definition of the sums.

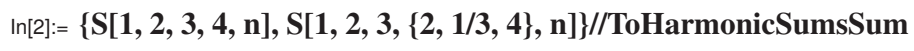

$\operatorname{Out}[2]=\left\{\sum_{\tau_{1}=1}^{n} \frac{\sum_{\tau_{2}=1}^{\tau_{1}} \frac{\sum_{\tau_{3}=1}^{\tau_{2}} \frac{\Sigma_{\tau_{4}=1}^{\tau_{3}} \frac{1}{\tau_{4}^{4}}}{\tau_{2}^{3}}}{\tau_{1}^{2}}}{\tau_{1}}, \sum_{\tau_{1}=1}^{n} \frac{2^{\tau_{1}} \sum_{\tau_{2}=1}^{\tau_{1}} \frac{3^{-\tau_{2}} \sum_{\tau_{3}=1}^{\tau_{2}} \frac{4^{\tau_{3}}}{\tau_{3}^{3}}}{\tau_{2}^{2}}}{\tau_{1}}\right\}$

$\operatorname{In}[3]:=\{\mathrm{S}[\{\{3,2,1\},\{4,1,2\},\{2,0,-2\}\}, n], S[\{\{3,2,1\},\{4,1,2\},\{2,0,2\}\},\{2,1 / 3,-4\}, n]\} / /$ ToHarmonicSumsSum 


$$
\text { Out[3] }=\left\{\sum_{\tau_{1}=1}^{n} \frac{\sum_{\tau_{2}=1}^{\tau_{1}} \frac{\sum_{\tau_{3}=1}^{\tau_{2}} \frac{(-1) \tau_{3}^{\tau_{3}}}{4 \tau_{3}^{2}}}{\left(1+4 \tau_{2}\right)^{2}}}{2+3 \tau_{1}}, \sum_{\tau_{1}=1}^{n} \frac{2^{\tau_{1}} \sum_{\tau_{2}=1}^{\tau_{1}} \frac{3^{-\tau_{2}} \sum_{\tau_{3}=1}^{\tau_{2}} \frac{(-1)^{\tau_{3}-1+\tau_{3}}}{\tau_{3}^{2}}}{\left(1+4 \tau_{2}\right)^{2}}}{2+3 \tau_{1}}\right\}
$$

Note that for internal reasons, sometimes the name CS is used to denote cyclotomic harmonic sums and cyclotomic S-sums.

$\ln [4]:=\{\operatorname{CS}[\{\{3,2,1\},\{4,1,2\},\{2,0,-2\}\}, n], \operatorname{CS}[\{\{3,2,1\},\{4,1,2\},\{2,0,2\}\},\{2,1 / 3,-4\}, n]\} / / T o H a r m o n i c S u m s S u m$

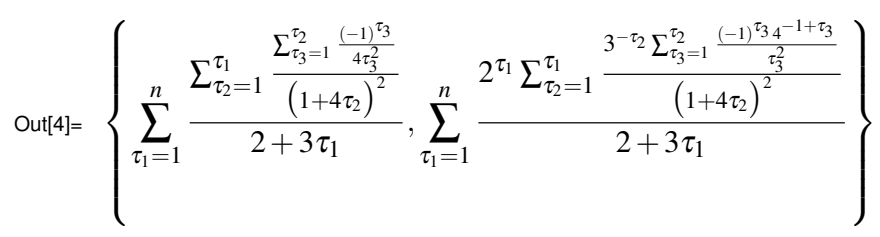

In addition HarmonicSums can deal with binomial sums, which are denoted by BS:

$\operatorname{In}[5]:=\operatorname{BS}[\{\{2,1,3\},\{1,0,1\}\},\{4,3\},\{\{\{2\},\{1,1\}\},\{\{1,1\},\{2\}\}\}, n] /$ ToHarmonicSumsSum

Out[5]= $\sum_{\tau_{1}=1}^{n} \frac{4^{\tau_{1}}\left(2 \tau_{1}\right) ! \sum_{\tau_{2}=1}^{\tau_{1}} \frac{3^{\tau_{2}}\left(\tau_{2} !\right)^{2}}{\left(2 \tau_{2}\right) ! \tau_{2}}}{\left(\tau_{1} !\right)^{2}\left(1+2 \tau_{1}\right)^{3}}$

Hence a summand of the form $\frac{x^{\tau_{i}}}{\left(a \tau_{i}+b\right)^{c}} \frac{\left(f_{1} \tau_{i}\right) ! \cdots\left(f_{j} \tau_{i}\right) !}{\left(g_{1} \tau_{i}\right) ! \cdots\left(g_{k} \tau_{i}\right) !}$ is represented by $\{a, b, c\}$ in the first, $x$ in the second and $\left\{\left\{f_{1}, \ldots, f_{j}\right\},\left\{g_{1}, \ldots, g_{k}\right\}\right\}$ in the third index set.

\section{Transformation to Nested Sums}

Using the command TransformToSSums an extension [8] of the algorithm described in [1] is performed to rewrite nested sum expressions in terms of harmonic sums, S-sums, cyclotomic harmonic sums and cyclotomic S-sums.

$$
\begin{aligned}
& \operatorname{In}[6]:=\sum_{i=1}^{n} \frac{2(5+2 i) \sum_{j=1}^{i} \frac{1}{j^{2}}}{\left(2+3 i+i^{2}\right)\left(12+7 i+i^{2}\right)} / / \text { TransformToSSums } \\
& \text { Out[6]= } \frac{1}{(n+1)(n+2)(n+3)(n+4)} \frac{1}{54}\left(-n\left(43 n^{3}+394 n^{2}+1163 n+1100\right)+36(n+1)^{2}\left(n^{2}+8 n+15\right) S_{2}(n)\right)
\end{aligned}
$$

\section{Definition of the Nested Integrals}

Harmonic polylogarithms, multiple polylogarithms and cyclotomic harmonic polylogarithms are denoted by the letter $\mathrm{H}$ as we can see in the following examples. The command ToHarmonicSums Integrate yields the definition of the nested integrals.

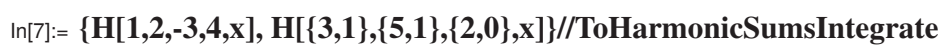

$$
\text { Out[] }=\left\{\int_{0}^{\mathrm{x}} \frac{\int_{0}^{\tau_{1}} \frac{\int_{0}^{\tau_{2}} \frac{\int_{0}^{\tau_{3}} \frac{1}{\tau_{4}-d} d \tau_{4}}{\tau_{3}+3} d \tau_{3}}{\tau_{2}-2} d \tau_{2}}{\tau_{1}-1} d \tau_{1}, \int_{0}^{\mathrm{x}} \frac{\tau_{1}\left(\int_{0}^{\tau_{1}} \frac{\tau_{2}\left(\int_{0}^{\tau_{2}} \frac{1}{\tau_{3}+1} d \tau_{3}\right)}{\tau_{2}^{4}+\tau_{2}^{3}+\tau_{2}^{2}+\tau_{2}+1} d \tau_{2}\right)}{\tau_{1}^{2}+\tau_{1}+1} d \tau_{1}\right\}
$$


Note that an index $a \in \mathbb{R}$ yields an iteration over $\frac{1}{\operatorname{sign}(a) \tau_{i}-a}$, while an index $\{l, k\} l, k \in \mathbb{N}$ indicates an iteration over $\frac{\tau_{i}^{k}}{\Phi_{l}\left(\tau_{i}\right)}$, where $\Phi_{l}$ is the $l$-th cyclotomic polynomial. For iterations over more general functions the name GL is reserved. The functions can then be defined using VarGL :

$\operatorname{In}[8]$ ]: $\mathbf{G L}\left[\left\{\sqrt{1-\operatorname{VarGL}}, \frac{1}{\operatorname{VarGL}+1}\right\}, \mathbf{x}\right] / /$ ToHarmonicSumsIntegrate

Out[8]= $\int_{0}^{x}\left(\int_{0}^{\tau_{1}} \frac{1}{1+\tau_{2}} d \tau_{2}\right) \sqrt{1-\tau_{1}} d \tau_{1}$

\section{Shuffle and Quasi-Shuffle Product}

The functions LinearExpand and LinearHExpand are provided to expand products of harmonic sum, S-sums, cyclotomic harmonic sums and cyclotomic S-sums and products of harmonic polylogarithms, multiple polylogarithms and cyclotomic harmonic polylogarithms, respectively.

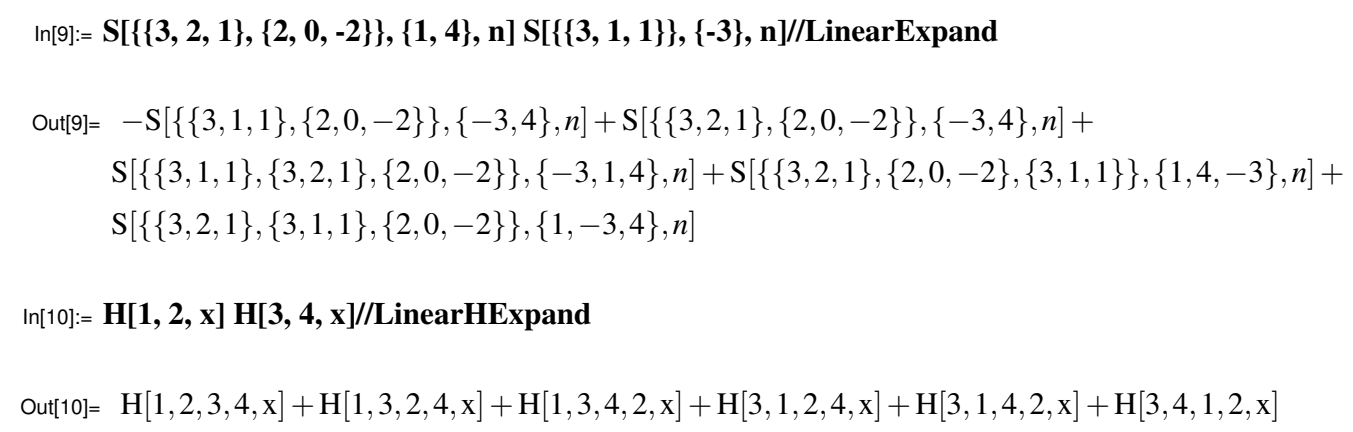

\section{Differentiation of Nested Sums}

In order to differentiate expressions involving harmonic sums, S-sums or cyclotomic harmonic sums the Mathematica function $D$ is extended. Note that here we actually work with the analytic continuation of these sums; for details see e.g., $[1,4,18,19]$.

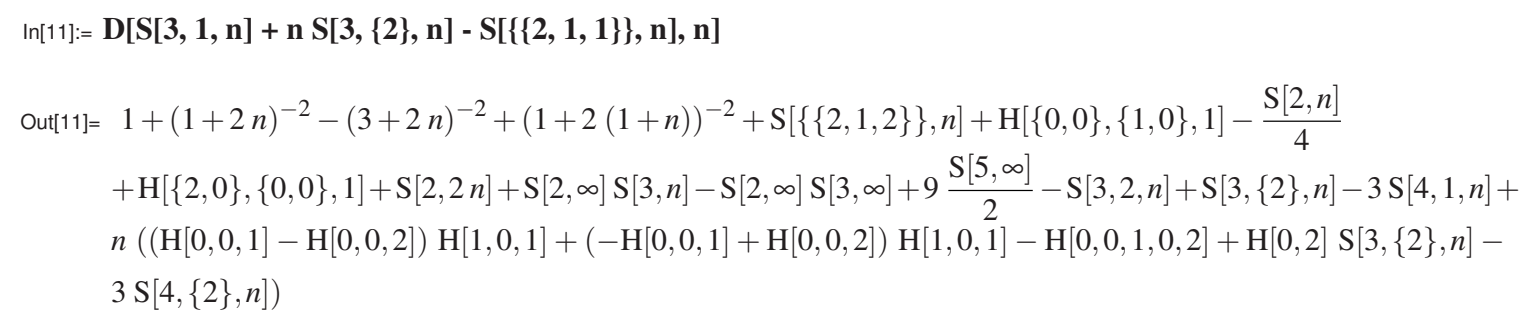

\section{Basis Representations}

For computing basis representations of harmonic sums, S-sums, cyclotomic harmonic sums, harmonic polylogarithms, cyclotomic polylogarithms or multiple polylogarithms HarmonicSums provides the functions ComputeHSumBasis, ComputessumBasis, ComputeCSumBasis and ComputeHLogBasis are provided. 
- ComputeHSumBasis $[\mathrm{w}, \mathrm{n}]$ computes a basis and the corresponding relations for harmonic sums at weight $w$. With the options UseDifferentiation and UseHalfInteger it can be specified whether relations due to differentiation and argument duplication should be used.

- ComputeSSumBasis $[\mathrm{w}, \mathrm{x}, \mathrm{n}]$ computes a basis and the corresponding relations for Ssums at weight $w$ where the allowed " $x$ " - indices are defined in the list $\mathrm{x}$. With the options UseDifferentiation and UseHalfInteger it can be specified whether relations due to differentiation and argument duplication should be used.

- ComputeCSumBasis [w, let, n] computes a basis and the corresponding relations for cyclotomic harmonic sums at weight $w$ with letters let. With the options UseDifferentiation, UseMultipleInteger and UseHalfInteger it can be specified whether relations due to differentiation and argument multiplication should be used.

- ComputehLogBasis $[\mathrm{w}, \mathrm{n}]$ computes a basis and the corresponding relations for multiple polylogarithms at weight $\mathrm{w}$. The option Alphabet->a and IndexStructure->i can be used to specify an alphabet or a special index structure respectively.

$\ln [12]$ ]: ComputeCSumBasis[2, $\{\{2,1\}\}, \mathbf{n}$, UseDifferentiation $->$ False,

UseMultipleInteger $->$ False, UseHalfInteger $->$ False

$$
\begin{aligned}
\text { Out[12] }= & \{\{\mathrm{S}[\{\{2,1,-2\}\}, n], \mathrm{S}[\{\{2,1,2\}\}, n], \mathrm{S}[\{\{2,1,-1\},\{2,1,1\}\}, n]\}, \\
& \left\{\mathrm{S}[\{\{2,1,1\},\{2,1,1\}\}, n] \rightarrow \frac{1}{2} \mathrm{~S}[\{\{2,1,1\}\}, n]^{2}+\frac{1}{2} \mathrm{~S}[\{\{2,1,2\}\}, n],\right. \\
& \mathrm{S}[\{\{2,1,1\},\{2,1,-1\}\}, n] \rightarrow \mathrm{S}[\{\{2,1,-2\}\}, n]+\mathrm{S}[\{\{2,1,-1\}\}, n] \mathrm{S}[\{\{2,1,1\}\}, n] \\
& -\mathrm{S}[\{\{2,1,-1\},\{2,1,1\}\}, n], \mathrm{S}[\{\{2,1,-1\},\{2,1,-1\}\}, n] \rightarrow \frac{1}{2} \mathrm{~S}[\{\{2,1,-1\}\}, n]^{2}+ \\
& \left.\left.\frac{1}{2} \mathrm{~S}[\{\{2,1,2\}\}, n]\right\}\right\}
\end{aligned}
$$

In order to look for relations for harmonic sums, S-sums and cyclotomic harmonic sums at infinity we can use the functions ComputeHSumInfBasis, ComputeSSumInfBasis and ComputeCSumInfBasis while for looking for relations between multiple polylogarithms and cyclotomic polylogarithms at 1 the functions ComputeGeneralizedH1Basis and ComputeCyclotomicH1Basis are provided.

$\ln [13]$ := ComputeCSumInfBasis[2, $\{\{2,1\}\}]$

$$
\begin{aligned}
\text { Out[13]= } & \{\{S[\{\{2,1,-2\}\}, \infty], S[\{\{2,1,2\}\}, \infty], S[\{\{2,1,-1\},\{2,1,1\}\}, \infty]\}, \\
& \left\{S[\{\{2,1,1\},\{2,1,1\}\}, \infty] \rightarrow \frac{1}{2} S[\{\{2,1,1\}\}, \infty]^{2}+\frac{1}{2} S[\{\{2,1,2\}\}, \infty],\right. \\
& S[\{\{2,1,1\},\{2,1,-1\}\}, \infty] \rightarrow S[\{\{2,1,-2\}\}, \infty]+S[\{\{2,1,-1\}\}, \infty] S[\{\{2,1,1\}\}, \infty] \\
& -S[\{\{2,1,-1\},\{2,1,1\}\}, \infty], S[\{\{2,1,-1\},\{2,1,-1\}\}, \infty] \rightarrow \frac{1}{2} S[\{\{2,1,-1\}\}, \infty]^{2}+ \\
& \left.\left.\frac{1}{2} S[\{\{2,1,2\}\}, \infty]\right\}\right\}
\end{aligned}
$$

For harmonic sums and cyclotomic harmonic sums tables with relations are provided [3, 9]. These tables can be applied using the command ReduceToBasis. With the options UseDiff- 
erentiation and UseHalfInteger it is possible to specify whether relations due to differentiation and argument duplication should be used.

ReduceToBasis [expr,n, Dynamic->True] computes relations for harmonic sums, $\mathrm{S}$-sums and cyclotomic harmonic sums in expr from scratch and applies them while ReduceToBasis [expr, n, Dynamic->Automatic] uses the precomputed tables and computes relations that exceed the tables form scratch.

ReduceToHBasis uses precomputed tables with relations between harmonic polylogarithms and applies them to expressions involving harmonic polylogarithms similar as for ReduceToBasis the options Dynamic->Automatic/True can be set.

ReduceConstants uses precomputed tables with relations between harmonic polylogarithms at argument 1 and harmonic sums at infinity to reduce the appearing constants as far as possible again the options Dynamic->Automatic/True can be set.

$\ln [14]=:$ ReduceToBasis $[S[2,1, n]+S[1,2, n], n]$

Out[14]= $\mathrm{S}[1, n] \mathrm{S}[2, n]+\mathrm{S}[3, n]$

$\ln [15]:=$ ReduceToBasis $[\mathrm{S}[5,5,\{3,3\}, \mathrm{n}], \mathrm{n}$, Dynamic $->$ True $]$

Out[15]= $\frac{1}{2}\left(\mathrm{~S}[5,\{3\}, n]^{2}+\mathrm{S}[10,\{9\}, n]\right)$

$\ln [16]:=$ ReduceToHBasis $[H[1,0, x]+H[0,1, x]]$

Out[16]= $H[0, x] H[1, x]$

$\ln [1]]:=$ ReduceConstants $[S[1,1,1,1,1,1,1,1, \infty]+2$ H[1, 0, 1] + H[0, 1, -1, 1], Dynamic -> Automatic]

Out[17]= $\frac{5}{201600}\left(\mathrm{~S}[1, \infty]^{8}+140 \mathrm{~S}[2, \infty] \mathrm{S}[1, \infty]^{6}+560 \mathrm{~S}[3, \infty] \mathrm{S}[1, \infty]^{5}+1890 \mathrm{~S}[2, \infty]^{2} \mathrm{~S}[1, \infty]^{4}\right.$

$+1120(5 \mathrm{~S}[2, \infty] \mathrm{S}[3, \infty]+6 \mathrm{~S}[5, \infty]) \mathrm{S}[1, \infty]^{3}+20\left(549 \mathrm{~S}[2, \infty]^{3}+280 \mathrm{~S}[3, \infty]^{2}\right) \mathrm{S}[1, \infty]^{2}$

$+720\left(21 \mathrm{~S}[3, \infty] \mathrm{S}[2, \infty]^{2}+28 \mathrm{~S}[5, \infty] \mathrm{S}[2, \infty]+40 \mathrm{~S}[7, \infty]\right) \mathrm{S}[1, \infty]+7893 \mathrm{~S}[2, \infty]^{4}$

$\left.-5600 \mathrm{~S}[2, \infty]\left(-\mathrm{S}[3, \infty]^{2}+54 \mathrm{~S}[-1, \infty]+72\right)+1680(8 \mathrm{~S}[3, \infty](\mathrm{S}[5, \infty]-15)+15 \mathrm{~S}[8, \infty])\right)$

\section{Series Expansions}

The function HarmonicSums Series [expr, $\{n, p, o r d\}]$ can be used to compute series expansions about the point $\mathrm{n}=\mathrm{p}$ of expressions expr involving harmonic sums, S-sums, cyclotomic harmonic sums, harmonic polylogarithms, multiple polylogarithms and cyclotomic harmonic polylogarithms up to a specified order ord.

$\ln [18]:=$ HarmonicSumsSeries $[n * S[2, n]+n * H[-2, n],\{n, 0,4\}] / /$ ReduceConstants

Out[18]= $n^{4}\left(4 \mathrm{z} 5+\frac{1}{24}\right)+n^{3}\left(-\frac{6 \mathrm{z}^{2}}{5}-\frac{1}{8}\right)+n^{2}\left(2 \mathrm{z} 3+\frac{1}{2}\right)$

$\ln [19]==$ HarmonicSumsSeries[n*S[2, n] + n*H[-2, n], $\{n, \infty, 4\}] / /$ ReduceConstants

Out[19] $=-n \mathrm{H}[0,2]+n \mathrm{H}[0, n]-\frac{4}{n^{3}}+\frac{5}{2 n^{2}}+n \mathrm{z} 2-\frac{3}{2 n}+1$ 
$\ln [20]:=$ HarmonicSumsSeries[S[3, 1, $\{1 / 2,1 / 3\}, \mathbf{n}],\{\mathrm{n}, \infty, 3\}]$

$$
\begin{aligned}
\text { Out[20] }= & \mathrm{S}\left[1,\left\{\frac{1}{3}\right\}, \infty\right]\left(-\mathrm{S}\left[3,\left\{\frac{1}{6}\right\}, \infty\right]+\mathrm{S}\left[3,\left\{\frac{1}{2}\right\}, \infty\right]+6^{-n}\left(\frac{1}{5 n^{3}}-\frac{3^{n}}{n^{3}}\right)\right)+ \\
& 6^{-n}\left(\frac{12}{25 n^{3}}-\frac{1}{5 n^{2}}\right) \mathrm{S}\left[2,\left\{\frac{1}{3}\right\}, \infty\right]+6^{-n}\left(\frac{42}{125 n^{3}}-\frac{6}{25 n^{2}}+\frac{1}{5 n}\right) \mathrm{S}\left[3,\left\{\frac{1}{3}\right\}, \infty\right]+ \\
& \mathrm{S}\left[2,\left\{\frac{1}{6}\right\}, \infty\right] \mathrm{S}\left[2,\left\{\frac{1}{3}\right\}, \infty\right]-\mathrm{S}\left[1,\left\{\frac{1}{6}\right\}, \infty\right] \mathrm{S}\left[3,\left\{\frac{1}{3}\right\}, \infty\right]-\mathrm{S}\left[4,\left\{\frac{1}{3}\right\}, \infty\right]+\mathrm{S}\left[1,3,\left\{\frac{1}{6}, 2\right\}, \infty\right]+ \\
& 6^{-n}\left(\frac{1}{5 n^{2}}-\frac{12}{25 n^{3}}\right) \mathrm{H}[0,3,1]+6^{-n}\left(-\frac{42}{125 n^{3}}+\frac{6}{25 n^{2}}-\frac{1}{5 n}\right) \mathrm{H}[0,0,3,1]-\frac{\mathrm{H}[3,1] 6^{-n}}{5 n^{3}}
\end{aligned}
$$

Note that $z 2, z 3, \ldots$ are used as abbreviations for $S[2, \infty], S[3, \infty], \ldots$ respectively. In order to compute asymptotic expansions of an $\mathrm{S}$-sums $\mathrm{S}\left[a_{1}, a_{2}, \ldots,\left\{x_{1}, x_{2}, \ldots\right\}, n\right]$ with $\left|x_{i}\right|>1$ for at least one $i$ the option PrincipalValue $->$ True has to be set:

$\ln [21]:=$ HarmonicSumsSeries[S[1,1,\{2,1\},n],\{n, $\infty, 3\}]$

Out[21] $=\mathrm{S}[1,1,\{2,1\}, n]$

$\ln [22]:=$ HarmonicSumsSeries[S[1,1,\{2,1\},n],\{n, $\infty, 3\}$, PrincipalValue->True]

Out[22] $=\frac{1}{2}\left(2^{n}\left(-\frac{2}{n^{2}}-\frac{43}{3 n^{3}}\right)-\frac{\pi^{2}}{2}+2^{n}\left(\frac{4}{n}+\frac{4}{n^{2}}+\frac{12}{n^{3}}\right) \mathrm{LG}[n]\right)$

Note that the function LG is defined as $\operatorname{LG}[n]:=\log (n)+\gamma$, where $\gamma$ is the Euler-Mascheroni constant. For computing asymptotic expansions of expressions of the form $\int_{0}^{1} x^{n} \mathrm{GL}[a, x] d x$ for $n \rightarrow \infty$ the command GLExpansion is provided:

$\ln [23]:=$ GLExpansion $[\mathbf{G L}[\{\sqrt{1+\operatorname{VarGL}}\}, \mathbf{x}], \mathbf{x}, \mathbf{n}, \mathbf{o r d}]$

Out[23] $=-\frac{2}{3 n^{5}}+\frac{4225}{96 \sqrt{2} n^{5}}+\frac{2}{3 n^{4}}-\frac{469}{24 \sqrt{2} n^{4}}-\frac{2}{3 n^{3}}+\frac{55}{6 \sqrt{2} n^{3}}+\frac{2}{3 n^{2}}-\frac{7 \sqrt{2}}{3 n^{2}}-\frac{2}{3 n}+\frac{4 \sqrt{2}}{3 n}$

The function HTOS can be used to compute the full power series expansions of harmonic polylogarithms, multiple polylogarithms and cyclotomic harmonic polylogarithms about 0 . STOH is used to perform the reverse direction.

$\operatorname{In}[24]:=\operatorname{HTOS}[\{H[-1,0,-1, \mathbf{x}], \mathbf{H}[-3,0,-1 / 2, x]\}]$

Out[24] $=\left\{\sum_{l_{1}=1}^{\infty} \frac{\mathrm{S}\left[2, l_{1}\right](-\mathrm{x})^{l_{1}}}{l_{1}}-\sum_{l_{1}=1}^{\infty} \frac{(-\mathrm{x})^{l_{1}}}{l_{1}^{3}}, \sum_{l_{1}=1}^{\infty} \frac{3^{-l_{1}}(-\mathrm{x})^{l_{1}} \mathrm{~S}\left[2,\{6\}, l_{1}\right]}{l_{1}}-\sum_{l_{1}=1}^{\infty} \frac{2^{l_{1}}(-\mathrm{x})^{l_{1}}}{l_{1}^{3}}\right\}$

$\operatorname{In}[25]:=\operatorname{SToH}\left[\left\{\sum_{l_{1}=1}^{\infty} \frac{(-x)^{l_{1}} \mathrm{~S}\left[6, l_{1}\right]}{l_{1}}, \sum_{l_{1}=1}^{\infty} \frac{3^{-l_{1}}(-x)^{l_{1}} \mathrm{~S}\left[2,\{6\}, l_{1}\right]}{l_{1}}\right\}\right]$

Out[25] $=\left\{\mathrm{H}[-1,0,-1, \mathrm{x}]-\mathrm{H}[0,0,-1, \mathrm{x}], \mathrm{H}\left[-3,0,-\frac{1}{2}, \mathrm{x}\right]-\mathrm{H}\left[0,0,-\frac{1}{2}, \mathrm{x}\right]\right\}$

For the more general iterated integrals GL the command GLTOS has to be used, note that this command internally relies on the recurrence solver of the package Sigma [24, 25].

$\operatorname{In}[26]:=\operatorname{GLToS}[\mathbf{G L}[\{\sqrt{4-\operatorname{VarGL}} \sqrt{\operatorname{VarGL}}\}, \mathbf{x}]]$ 
Out[26] $=\sum_{\mathrm{O}_{1}=2}^{\infty}-\frac{256 x^{\frac{1}{2}\left(-1+2 \mathrm{o}_{1}\right)}\left(\prod_{l_{1}=1}^{\mathrm{O}_{1}} \frac{-1+2 l_{1}}{8 \mathrm{l}_{1}}\right)\left(-1+\mathrm{o}_{1}\right) \mathrm{o}_{1}}{\left(-5+2 \mathrm{o}_{1}\right)\left(-3+2 \mathrm{o}_{1}\right)\left(-1+2 \mathrm{o}_{1}\right)^{2}}$

\section{Mellin Transformation and Inverse Mellin Transformation}

To compute the Mellin transform of a possibly weighted harmonic polylogarithm, multiple polylogarithm (with indices in $\mathbb{R} \backslash(-1,1) \cup\{0\}$ ) and cyclotomic polylogarithm $\mathrm{h} l \circ g[\mathrm{x}]$ we can use the command Mellin $[h \log [\mathrm{x}], \mathrm{x}, \mathrm{n}]$.

$\ln [27]:=\operatorname{Mellin}[H[1,0, x] /(1+x)+H[3, x] /(3-x), x, n]$

Out[27] $=-2(-1)^{n} \mathrm{~S}[3, \infty]+(-1)^{n} \mathrm{~S}[-2,-1, \infty]+(-1)^{n} \mathrm{~S}[-1,-2, \infty]-(-1)^{n} \mathrm{~S}[-1,2, n]+3^{n} \mathrm{~S}[1, n] \mathrm{S}[1,\{1 / 3\}, \infty]-$ $3^{n} \mathrm{~S}[1,\{1 / 3\}, n] \mathrm{S}[1,\{1 / 3\}, \infty]-3^{n} \mathrm{~S}[2,\{1 / 3\}, \infty]+3^{n} \mathrm{~S}[1,1,\{1 / 3,1\}, \infty]-3^{n} \mathrm{~S}[1,1,\{1,1 / 3\}, n]$

For computing the Mellin transform of more general input expr the command GeneralMellin $[\operatorname{expr}, \mathrm{x}, \mathrm{n}]$ is provided. Note that this command internally relies on the recurrence solver of the package Sigma.

$\ln [28]$ := GeneralMellin[InvMellGen[S[1, 2, $\{2,1 / 2\}, n]+S[1, n], n, x], n, x]$

$$
\begin{aligned}
\text { Out[28] }= & \frac{4(-1)^{n} \sqrt{2 \pi} n !}{(2 n+1)(2 n+3)(2 n+5)\left(n-\frac{1}{2}\right) !} \operatorname{BS}[\{\{1,0,0\}\},\{-(1 / 4)\},\{\{\{2\},\{1,1\}\}\}, n] \\
& +\frac{2\left(3(-5+6 \sqrt{2})+2(-8+13 \sqrt{2}) n+(-4+8 \sqrt{2}) n^{2}\right)}{3(n+1)(2 n+3)(2 n+5)} \\
& +\frac{(-1)^{n} \sqrt{\pi} n !}{(2 n+1)(2 n+3)(2 n+5)\left(n-\frac{1}{2}\right) !}(8 \sqrt{2}-15 \mathrm{GL}[\{\operatorname{VarGL} \sqrt{1+\operatorname{VarGL}}\}, 1]) \\
& +\frac{-\left(-1+3^{n+1}\right)(n+1) \mathrm{GL}\left[\left\{\frac{1}{3-\operatorname{VarGL}}\right\}, 1\right]+3^{n+1}(n+1) S_{1}\left(\frac{1}{3}, n\right)+1}{(n+1)^{2}}
\end{aligned}
$$

To compute the inverse Mellin transform of a harmonic sum or a S-sum denoted by sum we can use the command InvMellin [sum, $n, x]$. For a definition of S-sum we refer to [1]. Note that $\delta_{1-x}$ denotes the Dirac- $\delta$-distribution $\delta(1-x) \in D^{\prime}[0,1]$. For cyclotomic harmonic sums and S-sums which are not S-sums InvMellin yields an integral representations, where Mellin $[\mathrm{a}[\mathrm{x}, \mathrm{n}]]:=\int_{0}^{1} a(x, n) d x$ and Mellin $[\mathrm{a}[\mathrm{x}, \mathrm{n}],\{\mathrm{x}, \mathrm{c}, \mathrm{d}\}]:=\int_{c}^{d} a(x, n) d x$.

$\operatorname{In}[29]:=\operatorname{InvMellin}[\mathbf{S}[\mathbf{1}, \mathbf{2}, \mathbf{n}], \mathbf{n}, \mathbf{x}]$

Out[29] $=\frac{\mathrm{H}[1,0, \mathrm{x}]}{1-\mathrm{x}}$

$\operatorname{In}[30]:=\operatorname{InvMellin}[S[1,2,\{1,1 / 3\}, n], n, x]$

Out[30] $=\delta_{1-x}(-\mathrm{S}[1,\{1 / 3\}, \infty] \mathrm{S}[2,\{1 / 3\}, \infty]-2 \mathrm{~S}[3,\{1 / 3\}, \infty]+\mathrm{S}[1,2,\{1 / 3,1\}, \infty]+$ $\mathrm{S}[2,1,\{1 / 3,1\}, \infty])+\frac{3^{-n} \mathrm{~S}[2,\{1 / 3\}, \infty]}{3-\mathrm{x}}-\frac{\mathrm{S}[2,\{1 / 3\}, \infty]}{1-\mathrm{x}}-\frac{3^{-n} \mathrm{H}[3,0, \mathrm{x}]}{\mathrm{x}-3}$

$\operatorname{In}[31]:=\operatorname{InvMellin}[S[\{\{3,1,2\}\}, \mathbf{n}], \mathbf{n}, \mathbf{x}]$

$$
\begin{aligned}
\text { Out }[31]= & - \text { Mellin }\left[\mathrm{x}^{3 n} \mathrm{H}[0, \mathrm{x}]\right]-\frac{1}{3} \operatorname{Mellin}\left[\frac{\left(\mathrm{x}^{3 n}-1\right) \mathrm{H}[0, \mathrm{x}]}{\mathrm{x}-1}\right]+\frac{1}{3}\left(2 \operatorname{Mellin}\left[\frac{\left(\mathrm{x}^{3 n}-1\right) \mathrm{H}[0, \mathrm{x}]}{\mathrm{x}^{2}+\mathrm{x}+1}\right]+\right. \\
& \text { Mellin } \left.\left[\frac{\mathrm{x}\left(\mathrm{x}^{3 n}-1\right) \mathrm{H}[0, \mathrm{x}]}{\mathrm{x}^{2}+\mathrm{x}+1}\right]\right)-1
\end{aligned}
$$


In order to compute an integral representation of an expression expr containing general Ssums together with harmonic sums and cyclotomic sums the command InvMel lGen [ expr, $\mathrm{n}, \mathrm{x}$ ] can be used:

In[32]:= CollectMellinGen[InvMellGen[S[1, 2, $\{2,1 / 2\}, n]+S[1, n], n, x], n, x]$

Out[32] $=$ MellinGen $\left[-\frac{\mathrm{H}[2,0,1]\left(x^{n}-1\right)}{x-1},\{x, 1,2\}\right]+$ MellinGen $\left[\frac{\left(x^{n}-1\right)(1-\mathrm{H}[2,0, x])}{x-1},\{x, 0,1\}\right]$

Note that here we use the notation MellinGen $[\mathrm{a}[\mathrm{x}, \mathrm{n}],\{\mathrm{x}, \mathrm{c}, \mathrm{d}\}]:=\int_{c}^{d} a(x, n) d x$.

\section{Conclusion}

In this paper we summarized some features of the computer algebra package HarmonicSums . Due to space limitations we had to restrict to the presentation of the main commands, while there are many more commands implemented. For more information we refer to [1,2,4]. The package together with several precomputed tables and a more detailed description can be downloaded at http://www.risc.jku.at/research/combinat/software/HarmonicSums.

\section{References}

[1] J. Ablinger, Computer Algebra Algorithms for Special Functions in Particle Physics, $\mathrm{PhD}$ thesis, J. Kepler University Linz, [math-ph/1305.0687], 2012.

[2] J. Ablinger, A Computer Algebra Toolbox for Harmonic Sums related to Particle Physics, Diploma thesis, J. Kepler University Linz, [math-ph/1011.1176], 2009.

[3] J. Ablinger, J. Blümlein and C. Schneider, Harmonic sums and polylogarithms generated by cyclotomic polynomials, J. Math. Phys. 52, [hep-ph/1007.0375], 2011.

[4] J. Ablinger, J. Blümlein and C. Schneider, Analytic and Algorithmic Aspects of Generalized Harmonic Sums and Polylogarithms, J. Math. Phys. 54, [math-ph/1302.0378], 2013.

[5] J. Ablinger, J. Blümlein and C. Schneider, Generalized Harmonic, Cyclotomic, and Binomial Sums, their Polylogarithms and Special Numbers, J. Phys. Conf. Ser. 523, [math-ph/1310.5645], 2014.

[6] J. Ablinger and J. Blümlein, Harmonic Sums, Polylogarithms, Special Numbers, and their Generalizations, in: Computer Algebra in Quantum Field Theory: Integration, Summation and Special Functions, Texts \& Monographs in Symbolic Computation, Eds. C. Schneider and J. Blümlein, (Springer, Wien, 2013), [math-ph/1304.7071].

[7] J. Ablinger, J. Blümlein, C. Raab, and C. Schneider, Iterated Binomial Sums and their Associated Iterated Integrals, [hep-th/1407.1822], 2014.

[8] J. Ablinger and C. Schneider, in preparation.

[9] J. Ablinger, J. Blümlein and C. Schneider, in preparation.

[10] J. Ablinger, J. Blümlein, C. Raab, C. Schneider and F. Wissbrock, Calculating Massive 3-loop Graphs for Operator Matrix Elements by the Method of Hyperlogarithms, Nucl. Phys. B 885,

[hep-ph/1403.1137], 2014. 
[11] J. Ablinger, J. Blümlein, A. Hasselhuhn, S. Klein, C. Schneider and F. Wissbrock, Massive 3-loop Ladder Diagrams for Quarkonic Local Operator Matrix Elements, Nucl. Phys. B 864, [hep-ph/1206.2252v1], 2012.

[12] J. Ablinger, J. Blümlein, A. De Freitas, A. Hasselhuhn, A. von Manteuffel, M. Round and C. Schneider, The $O\left(\alpha_{s}^{3} T_{F}^{2}\right)$ Contributions to the Gluonic Operator Matrix Element, Nucl. Phys. B 885, [hep-ph/1405.4259], 2014.

[13] J. Ablinger, J. Blümlein, A. De Freitas A. Hasselhuhn, A. von Manteuffel, M. Round, C. Schneider and F. Wissbrock, The Transition Matrix Element $A_{g q}(N)$ of the Variable Flavor Number Scheme at $O\left(\alpha_{s}^{3}\right)$, Nucl. Phys. B 882, [hep-ph/1402.0359], 2014.

[14] J. Ablinger, A. Behring, J. Blümlein, A. De Freitas, A. Hasselhuhn, A. von Manteuffel, M. Round, C. Schneider, and F. Wißbrock, The 3-Loop Non-Singlet Heavy Flavor Contributions and Anomalous Dimensions for the Structure Function $F_{2}\left(x, Q^{2}\right)$ and Transversity, [hep-ph/1406.4654], 2014.

[15] J. Blümlein and S. Kurth, Harmonic sums and Mellin transforms up to two-loop order. Phys. Rev. D 60 014018, [hep-ph/9810241v2], 1999.

[16] J. Blümlein, Algebraic relations between harmonic sums and associated quantities, Comput. Phys. Commun. 159, [hep-ph/0311046v1], 2004.

[17] J. Blümlein, Structural Relations between Nested Harmonic Sums, Nucl. Phys. B (Proc. Suppl.) 183, 2008.

[18] J. Blümlein, Structural relations of harmonic sums and Mellin transforms up to weight w=5, Comput. Phys. Commun. 180, [hep-ph/0901.3106], 2009.

[19] J. Blümlein, Structural relations of harmonic sums and Mellin transforms at weight w=6, Clay Mathematics Proceedings 10, [math-ph/0901.0837], 2010.

[20] J. Blümlein, D.J. Broadhurst and J.A.M. Vermaseren The Multiple Zeta Value data mine, Comput. Phys. Commun. 181, [math-ph/0907.2557v2], 2010.

[21] A.I. Davydychev, M.Yu. Kalmykov, Massive Feynman diagrams and inverse binomial sums, Nucl. Phys. B 699, [hep-ph/0303162v4], 2004.

[22] S. Moch, P. Uwer and S. Weinzierl, Nested sums, expansion of transcendental functions, and multiscale multiloop integrals, J. Math. Phys. 43, [hep-ph/0110083v2], 2002.

[23] E. Remiddi and J.A.M. Vermaseren, Harmonic Polylogarithms, Int. J. Mod. Phys. A15, [hep-ph/9905237v1], 2000.

[24] C. Schneider, Symbolic Summation Assists Combinatorics, Sem. Lothar. Combin 56, 2007.

[25] C. Schneider, Symbolic Summation in Difference Fields, PhD thesis, J. Kepler University Linz, 2001.

[26] J.A.M. Vermaseren, Harmonic sums, Mellin transforms and Integrals, Int. J. Mod. Phys. A14, [hep-ph/9806280v1], 1999.

[27] S. Weinzierl, Expansion around half-integer values, binomial sums and inverse binomial sums, J. Math. Phys. 45, [hep-ph/0402131v1], 2004. 